\title{
Design of Integrated Wireless Network for Rural India
}

\author{
Shashikant S. Hippargi \\ PhD Research Student \\ Department of Electronics and Telecommunication \\ Engineering \\ SVERI's C.O.E., Pandharpur, Maharastra State, \\ India
}

\begin{abstract}
India is known as an agricultural country and has a variety of land with different climatic conditions throughout the year. After understanding and analyzing the different physical parameters, implementation of a good strategy for crop cultivation is needed. To provide development in all regions it is very much needed to connect rural part with urban India. Wired technology is a costly affair and hence wireless technology can be thought for implementing this system. Due to a large geographical area and less income it is very difficult to setup a network for connectivity. GSM networks are available with limitations of range in rural part of India. Single network cannot serve this purpose of setting up a network with all signal availability to solve this problem. An integrated wireless network is designed to establish last mile connectivity to enhance the livelihoods of these rural areas. A sensor based network and integration of three most popular technologies using Bluetooth, Wi-Fi and GSM is combined to form an integrated network to sense and communicate the physical parameters of farm from anywhere anytime to any part of the world. Also, the server is created to store the parameters sensed by sensors which can be displayed on PDA using android app.
\end{abstract}

Keywords

PDA, GSM, Wi-Fi, NodeMCU, HC-05.

\section{INTRODUCTION}

Agriculture being the main occupation of rural India, innovation is needed to improve both the quality and quantity of crops. Around $70 \%$ of the population is dependent on agriculture and its related services. Due to lack of knowledge less water, low income, failure of crop now a days farmers are committing suicide. Statics states that more than 3lakh farmers have committed suicide since 1995 in India[1]. In the year 2014 around 5600 farmers committed suicide[2]. A sensing technology can be used and precision farming can be done [3]. For good quality and efficient production of any crop a detailed study of climatic condition such as temperature, humidity must be done. At the same time analysis of soil content such as Nitrogen, Phosphorus and Potassium (NPK) is needed. These parameters have to be communicated to the experts so that proper suggestions can be given to farmers to increase the yield of crop. Communication of required parameters from the farmer's field to researcher has to be done as and when needed. Sensors are connected at the farmers end and an integrated network is established between sensors to the area where network connectivity is available. In this paper a low cost solution is designed to cover the last mile connectivity.

\section{RELATED WORK}

A system is established using sensing elements used to monitor temperature, humidity, water level etc with

\author{
Shrikant K. Bodhe, PhD \\ Research Guide \\ Department of Electronics and Telecommunication \\ Engineering \\ SVERI's C.O.E., Pandharpur, Maharastra State, \\ India
}

\section{ARMEGA8535 and Zigbee module as a transceiver [4].}

A GSM based model is designed where real time monitoring of parameters like $\mathrm{pH}$, soil moisture is done and communicated through SMS to decide the quantity of fertilizer in rice production for real time monitoring [5].

Data is transmitted wirelessly to reduce the cable structure in fields to controlling centre through wireless Sensor Network using Zigbee [6].

A setup of Wireless Sensor Network is done to monitor and analyze the air quality. Later the analysis is presented in different formats as per the requirement of end user [7].

Sensor nodes are created to sense the physical parameters and communicate among themselves. Parameters like soil moisture and temperature is collected and analysis is done to reduce the water consumption as per requirement [8]

Agriculture Information Technology is the tool which is becoming more prominent for utilization of agriculture resources and helps in increasing the agricultural production. Proper information is collected and communicated through Agriculture Information Management to improve the efficiency of agricultural production [9].

Importance of Wireless Sensor Network with analysis of its drawback is quoted. An App is developed to record and analyze the sensed data as temperature, moisture etc. Depending on the analysis farmer is informed about the required crop to be cultivated [10].

A system is designed without wire having sensors to monitor parameters at any location. Zigbee protocol based solution is proposed. For larger distance addition of GSM or Wi-Fi transmitter may be done [11].

\section{IMPLEMENTATION}

\subsection{Overview}

A system is designed where the physical parameter like temperature, humidity or soil moisture is sensed by the different sensors as DH11 or FC-28 and communicated over the wireless integrated network. A Scenario is considered where a large area is covered with GSM connectivity and a data has to be sensed from an area which is beyond the GSM connectivity. An extension of connectivity is provided by integrating GSM network with two different networks as Wireless Fidelity (Wi-Fi) and Bluetooth network which acts as intermediate network to cover the required range from which the data is to be sensed.

In this system as shown in block diagram whole communication is done through 3 modules. First module consist of Sensor which is connected through ATmega 328 to Bluetooth device which is a Master and acts as a transmitter. A huge data sensed by the sensor is continuously transmitted which is received by another Bluetooth module connected to 
module 2. Module 2 which is a Node MCU takes the received data from Bluetooth module which is a slave, process it and transmit it through inbuilt Wi-Fi module. A hotspot is used to connect Module 2 and Module 3 through Wi-Fi connected in Master and Slave mode. This transmitted data is received by inbuilt Wi-Fi of Module 3 which is also a Node MCU and process it for the transmission through GSM module connected to Module 3 through ATmega 328. This transmitted data from GSM module can be received in any Personal Digital Assistant (PDA-for example Cell phone) at any corner of the world.

In this system, sensor takes large number of data within very small time. All these data are not required at the other end. So code based method is designed to capture the data whenever it is required. A code \#Temp is sent whenever the data is required by the user through PDA to module no. 3. After detecting this code the sensed data at that particular time is sent back to PDA.

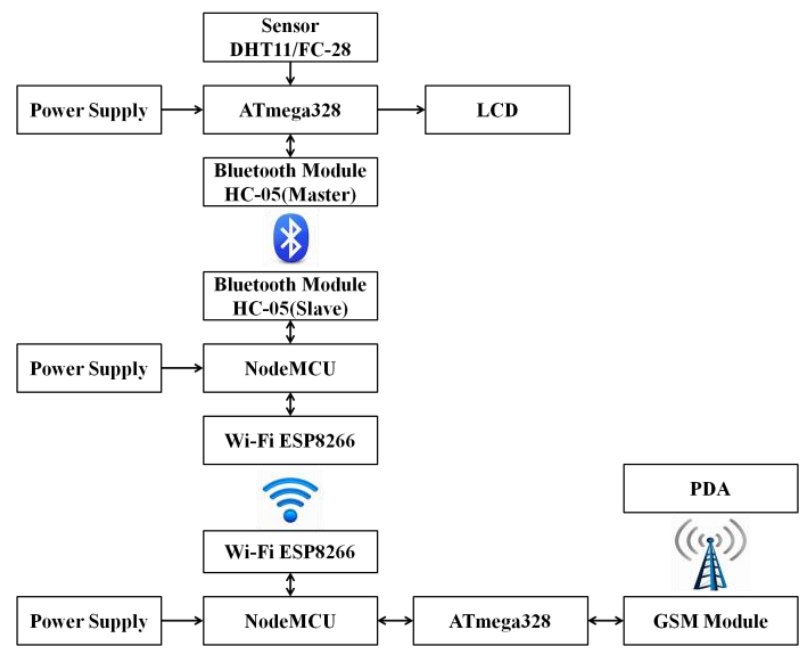

Fig 1: Block Diagram

\subsection{Selection of Hardwares}

Controller: NodeMCU (Node Micro Controller Unit) is an open source platform. It includes hardware with firmware for ESP8266 Wi-Fi SOC. It's a low cost device with Wi-Fi transceiver having ISM $2.4 \mathrm{GHz}$

Bluetooth Module: Easy to interface with controller via serial communication and required baud rate can be set. When in operation it requires $1.8 \mathrm{~V}$ with $3.3 \mathrm{~V}-5 \mathrm{~V}$ Input/Output. It can be configured using AT commands.

ESP8266: It have a 32 bit processor and supports $802.11 / \mathrm{b} / \mathrm{g} / \mathrm{n}$ wireless protocols. Its operating voltage is $2.5 \mathrm{~V}$ to $3.5 \mathrm{~V}$ and requires a very low power for transmission and reception. Easy configuration using AT commands and its operating frequency range is $2.4 \mathrm{GHz}$

SIM900A: It is a Dual band 900/1900 MHz GSM/GPRS module. A very low power of $18 \mathrm{~mW}$ is required during sleep mode with $1.5 \mathrm{~mA}$ of current. It is easy to configure using AT commands.

\subsection{Procedure to Set Modules for Communication}

3.3.1 Paring of Bluetooth in Master-Slave Mode

Two HC-05 modules have to set in Master-Slave mode to have direct communication as soon as the system is started. It is to be ensured that the baud rate of both Master and Slave devices must be same which is 9600 in this system. AT commands are used in serial monitor mode of Arduino IDE to set the Bluetooth module as master and slave mode

$\mathrm{AT}+\mathrm{ROLE}=1$ sets the Bluetooth device as Master

$\mathrm{AT}+\mathrm{ROLE}=0$ sets the Bluetooth device as Slave.

For these two devices to be connected in automatic mode commands to be executed on Master side are

$\mathrm{AT}+\mathrm{CMODE}=0$ for fix address

$\mathrm{AT}+\mathrm{BIND}=<$ address of the slave mode $>$

\subsubsection{Connection of Wi-Fi Devices in Server Client Mode}

Two Wi-Fi devices ESP8266 are connected in server-client mode using a cell phone hotspot. A fixed connection between server and client is done by setting SSID name and password using arrays of character on both server and client side. Following commands are used to set IP address of the server, gateway and subnet mask of used network

IP Address ip(192, 168, 0, 80);

IP Address gateway(192,168,0,1);

IP Address subnet $(255,255,255,0)$;

Once server client is connected status will be shown on cell phone as two devices connected. Baud rate for Wi-Fi to Wi-Fi communication is set to 115200 . Now, the communication can be started between server and client using Wi-Fi.

\subsubsection{GSM module with ATmega328}

A GSM module SIM900A is connected to NodeMCU through ATmega328 in text mode. A particular SIM card number located anywhere is used to get the sensed data through SMS when a code \#Temp is received by GSM module. Following commands are used to set the SIM card number to send and receive the message from module 3 .

$\mathrm{AT}+\mathrm{CMGF}=1$ set the GSM SIM900A in text mode

$\mathrm{AT}+\mathrm{CMGS}=\langle\mathrm{SIM}$ card number receive sensed data $>$

$\mathrm{AT}+\mathrm{CNMI}=2,2,0,0,0$ sets to receive live SMS

\subsection{Android Application for display of parameters}

Data received by the GSM module is saved in server using Apache xampp server. An android app is developed which displays the parameters sensed by the sensors on module 1 for easy understanding by the user having PDA.

\section{RESULTS}

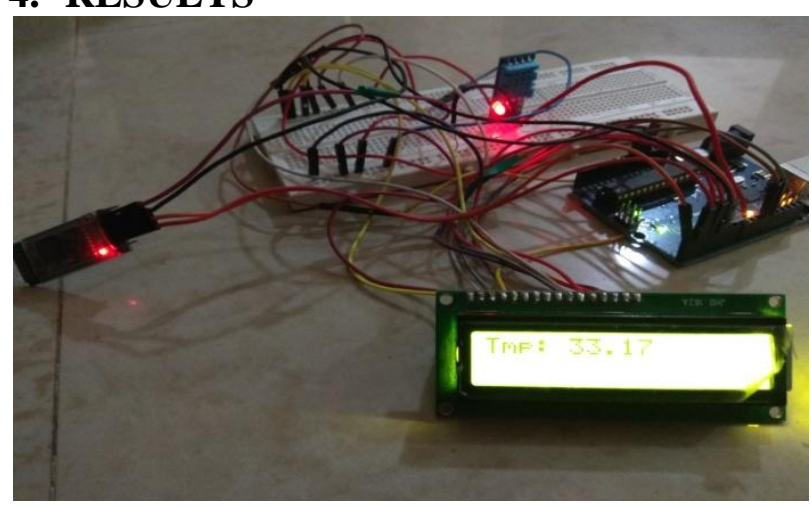

Fig 2: Module 1 
Figure 2 shows Module 1 in which sensor DHT11 and Bluetooth module HC-05 are connected to ATmega328. LCD connected to module 1 shows the current temperature sensed by the sensor and to be communicated to the user.

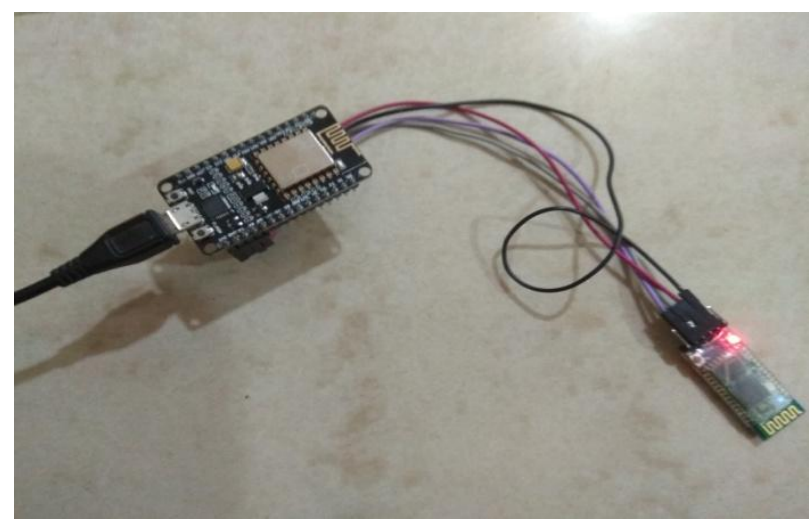

Fig 3: Module 2

Bluetooth module HC-05 is connected to NodeMCU as shown in figure 3 . The temperature sent by $\mathrm{HC}-05$ from module 1 is received and sent through ESP8266.

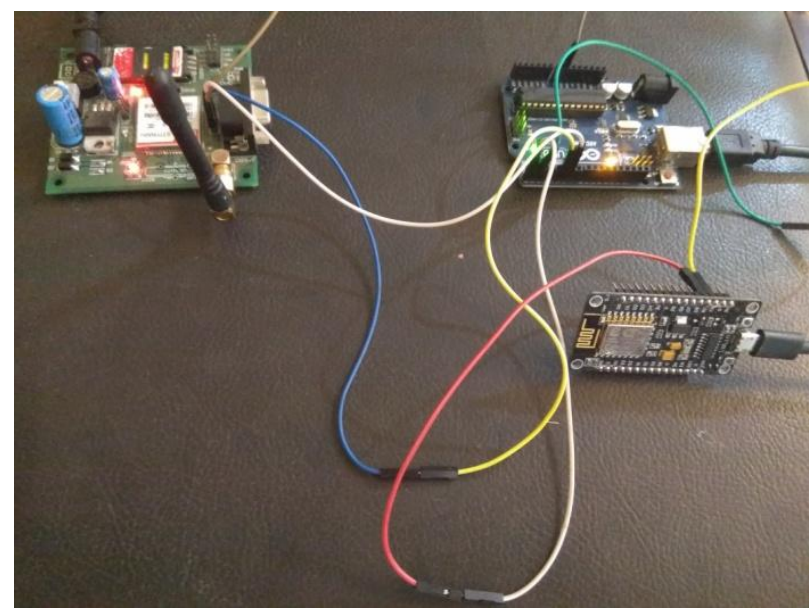

Fig 4: Module 3

Figure 4 shows module 3 which receives the temperature sent by module 2 by NodeMCU using ESP8266 Wi-Fi. SIM900A is interfaced with ATmega328 which is connected in parallel with NodeMCU. Temperature received by this module is sent to the user PDA (cell phone).

Figure 5 shows both the Wi-Fi devices from Module 2 and Module 3 connected to Hotspot.

4:43 PM
$<$ Connected devices
Limit of connected devices
Unlimited
Blocklist
View devices which aren't allowed to connect to this
hotspot
CONNECTED DEVICES
60:01:94:20:24:C4
ESP_76F8E1
5C:Cf:7f:76:f8:e1

Fig 5: Wi-Fi ESP8266 connected to Hotspot
Figure 6 shows the code \#Temp sent by the user to Module 3 which returns the temperature sensed by DHT11.

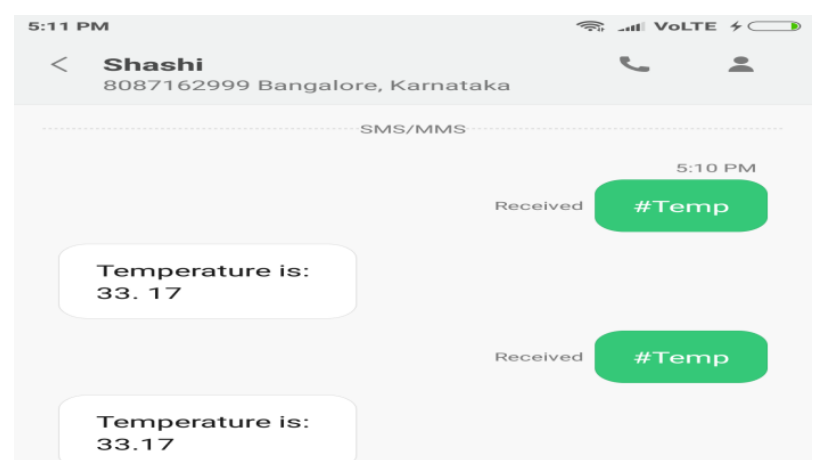

Fig 6: Temperature received at user side through SMS

Testing of both Bluetooth range and Wi-Fi range is done in open space to calculate the area covered by the whole system as shown in figure 7.

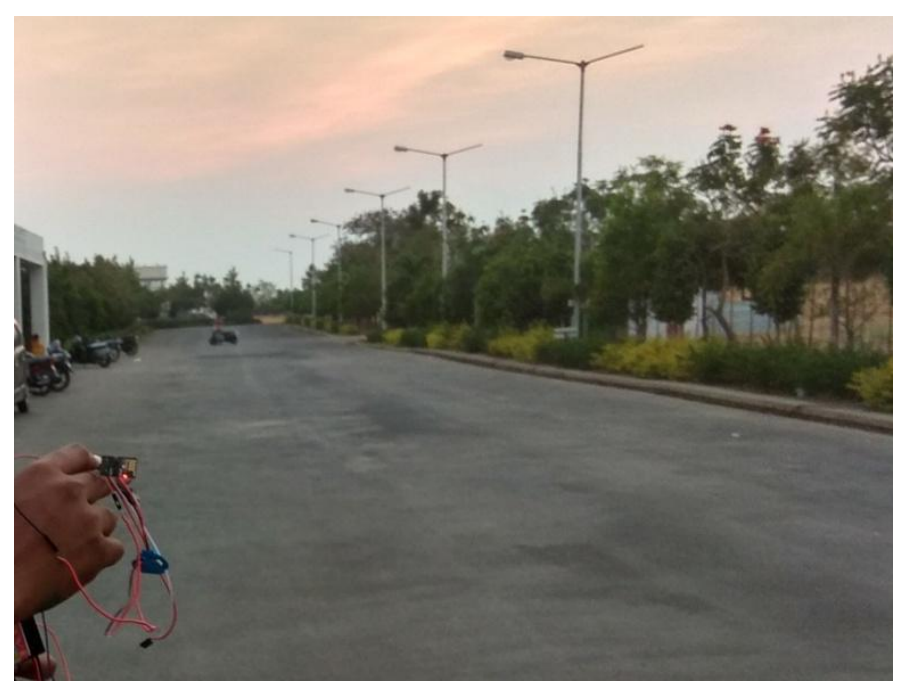

Fig 7: Testing of Wi-Fi Range

From the designed system a distance of $36 \mathrm{~m}$ for bluetooth to bluetooth and $350 \mathrm{~m}$ for $\mathrm{Wi}-\mathrm{Fi}$ to $\mathrm{Wi}-\mathrm{Fi}$ is covered. So, the total distance covered by both Module 1 and Module 2 is $380 \mathrm{~m}$.

If the Module 1 is repeated in the region covered by Module 2 as shown in figure 8,then a large area can be covered by sensors. Total area covered by repeating 19 in number of Module1 will be

Total radius covered will be $\mathrm{r}=175 \mathrm{~m}$

Area covered $\mathrm{A}=\pi \mathrm{r}^{2}$

$$
\begin{aligned}
& =3.14 *(175)^{2} \\
& =96162.5 \mathrm{~m}^{2}
\end{aligned}
$$

So, this system can sense approximately $\mathbf{1 0}$ hectare of farming land. 
Fig 8: Region covered by Module 1 and Module 2

Screen shots for registration, login and display of sensor readings are shown in figure 9 , figure 10 and figure 11 respectively.

\section{Fig 9: Screenshot for registration on Mobile phone}

The primary and essential step to start the process is registration. User is supposed to register only once by providing Name, Email, Contact, Password, Address and Pincode.

\section{Fig 10: Screenshot for $\log$ in by the user}

After completion of registration with the unique email id and password, user need to login to keep record of test cases.

Fig 11: Display of readings communicated over integrated wireless network using android app

Figure 11 shows the parameters sensed by different sensors at Module 1 on android app.

\section{CONCLUSION}

In this paper, a prototype of integrated network of Bluetooth, Wi-Fi, and GSM which is a low cost and low power system that makes last mile communication in rural area. This network will help to send the required parameters sensed by sensors from farms in rural India where GSM connectivity is limited. This system is a prototype to cover the area in meters depending upon the requirement. The range and area covered can be increased by selecting Bluetooth module HC-12, large range Wi-Fi hotspot to cover regions in kilo meters. Also, the app is developed to show the readings sensed by the sensors. 


\section{REFERENCES}

[1] https://yourstory.com/2016/10/farmer-suicides/

[2] National Crime Reports Bureau, ADSI Report Annual 2014 Government of India, p. 242, table 2.11

[3] Sreelakshmi, P., et al. "Automated Agrobot." Indian Journal of Science and Technology 9.30 (2016).

[4] Balaji Bhanu, Raghava Rao, J.V.N. Ramesh and Mohammed Ali hussain, "Agriculture Field Monitoring and Analysis using Wireless Sensor Networks for improving Crop Production”, 2014 Eleventh International Conference on Wireless and Optical Communications Networks (WOCN)

[5] S. Vijayakumar, J. Nelson Rosario, "Preliminary Design for Crop Monitoring Involving Water and Fertilizer Conservation Using Wireless Sensor Networks", Communication Software and Networks (ICCSN), 2011 IEEE 3rd International Conference.

[6] Zulhani Rasin, Hizzi Hamzah Mohd, Shahrieel Mohd Aras, "Application and Evaluation of High Power Zigbee Based Wireless Sensor Network in Water Irrigation Control Monitoring System", 2009 IEEE Symposium on Industrial Electronics and Applications (ISIEA 2009), October 4-6, 2009, Kuala Lumpur, Malaysia.
[7] Elias Yaacoub, Abdullah Kadri, Mohammed Mushtaha, and Adnan Abu-Dayya, "Air Quality Monitoring and Analysis in Qatar using a Wireless Sensor Network Deployment", 2013, 9th International Wireless Communications and Mobile Computing Conference (IWCMC).

[8] Nelson Sales, Orlando Remédios, Artur Arsenio, "Wireless Sensor and Actuator System for Smart Irrigation on the Cloud", Internet of Things (WF-IoT), 2015 IEEE 2nd World Forum.

[9] Duan Yan-e, "Design of Intelligent Agriculture Management Information System Based on IoT", 2011 Fourth International Conference on Intelligent Computation Technology and Automation.

[10] Varghese, Vijo T., Kalyan Sasidhar, and P. Rekha. "A status quo of WSN systems for agriculture." Advances in Computing, Communications and Informatics (ICACCI), 2015 International Conference on. IEEE, 2015.

[11] Nguyen Tang Kha Duy, Nguyen Dinh Tu, Tra Hoang Son, and Luong Hong Duy Khanh, "Automated Monitoring and Control System for Shrimp Farms Based on Embedded System and Wireless Sensor Network", Electrical, Computer and Communication Technologies (ICECCT), 2015 IEEE International Conference. 